\title{
Clinical profile of thrombocytopenia in pregnancy
}

\author{
Salil Barsode ${ }^{1 *}$, Vaishali Tarlekar ${ }^{2}$, Munaza Shora ${ }^{3}$, Savita Mehendale $^{4}$ \\ ${ }^{1}$ Associate Professor, ${ }^{2,4}$ Professor, ${ }^{3}$ Junior Resident, ${ }^{1-3}$ Dept. of Obstetrics and Gynaecology, ${ }^{1-4}$ Bharati Vidyapeeth Deemed University, \\ Medical College and Bharati Hospital, Pune, Maharashtra, India
}

Corresponding Author: Salil Barsode

Email: barsodes@gmail.com

Received: $18^{\text {th }}$ November, 2018

Accepted: $16^{\text {th }}$ May, 2019

\begin{abstract}
Thrombocytopenia is defined as platelet count of less than normal for the laboratory. This typically is at or very close to1.5 lakh/cumm. Hence platelet count of less than $1.5 \mathrm{lakh} / \mathrm{cumm}$ is called as thrombocytopenia. Thrombocytopenia in pregnancy is not an uncommon finding; in fact, it is the second most common hematological disorder in pregnancy after anaemia and affects nearly 6 to $15 \%$; on an average $10 \%$ of all pregnancies. The reported prevalence of maternal thrombocytopenia is variable. In a recent survey in 2000 involving 6770 pregnant women, prevalence of thrombocytopenia was found to be $11.6 \%$. A prospective observational study conducted to identify causes for thrombocytopenia in pregnancy along with its neonatal outcome Out of total, $30 \%$ patients delivered low birth weight babies and $70 \%$ delivered babies with birth wt of $>2.5 \mathrm{~kg}$. Occurrence of low birth weight babies in mothers with GT and ITP were comparable being $27.3 \%$ and $33.3 \%$ respectively. APGAR score (at $1 \mathrm{~min}$ ) of neonates was less than 7 in $12 \%$ neonates while at 5 min it was below 7 in only $1(2 \%)$ baby. Neonates born to mothers with ITP had poor 1 minute APGAR score when compared to neonates born to patients with GT. There was no significant relationship found between maternal and fetal platelet counts in patients with gestational thrombocytopenia. ITP leads to neonatal thrombocytopenia.
\end{abstract}

Keywords: Cord blood, Thrombocytopenia, APGAR scoring, ITP, Gestational thrombocytopenia.

\section{Introduction}

Platelet count of less than $1.5 \mathrm{lakh} / \mathrm{cumm}$ is called as thrombocytopenia. ${ }^{1,2}$ Thrombocytopenia in pregnancy is not an uncommon finding; in fact, it is the second most common hematological disorder in pregnancy after anaemia ${ }^{3}$ and affects nearly 6 to $15 \%$; on an average $10 \%$ of all pregnancies. ${ }^{1,2,4-6}$ Thrombocytopenia can be graded as mild (platelet count between 1lakh/cumm to 1.49 lakh/cumm), moderate (50,000/cumm-1lakh/cumm) and severe $(<50000 /$ cumm $){ }^{7}$ Severe degree can manifest as petechiae, ecchymoses, epistaxis, gingival bleed, hematuria and rarely as gastrointestinal bleed or intracranial hemorrhage (ICH) etc. However, mild and moderate degree of thrombocytopenia rarely manifest. Thrombocytopenia in pregnancy can occur from a variety of causes; physiological to pathological; ranging from benign disorders to life threatening ones. It can be due to either Gestational Thrombocytopenia (GT) or obstetric disorders like hypertensive disorders of pregnancy [preeclampsia, eclampsia \& its complications such as Hemolysis Elevated Liver enzymes and Low Platelets (HELLP)], amniotic fluid embolism, Disseminated Intravascular Coagulopathy (DIC) or medical causes like Thrombotic Thrombocytopenic Purpura (TTP)/Hemolytic Uremic Syndrome (HUS), autoimmune disorders:- Systemic Lupus Erythematosus (SLE), Idiopathic Thrombocytopenic Purpura (ITP), antiphospholipid syndrome, infections:- Human Immunodeficiency Virus (HIV), malaria, dengue, leptospirosis, sepsis, hypersplenism, primary bone marrow dysfunction:- leukemia, aplastic anemia, folate deficiency, congenital thrombocytopenia etc. It may also be spurious/pseudo thrombocytopenia or drug induced thrombocytopenia. ${ }^{1}$ Drugs causing thrombocytopenia are: antibiotics: (Ampicillin, Penicillin, Rifampicin), diuretics: (Thiazides, Furosemide), analgesics: (Aspirin, Acetaminophen, Indomethacin), anticonvulsants: (Phenytoin, Valproate, Carbamazepine) etc. ${ }^{1}$

Gestational Thrombocytopenia (GT) is a clinically benign thrombocytopenic disorder usually occurring in late pregnancy, with mild to moderate asymptomatic decrease in platelet counts. There is no previous history of any autoimmune disease, it resolves spontaneously after delivery and is usually of limited obstetric and hematological significance. ${ }^{1,2,10-13}$ However, prevalence of ITP is $\sim 100$ times less than that of GT, but it is one of the common causes of significant thrombocytopenia in first trimester. A history of prior thrombocytopenia, underlying autoimmune disease or severe thrombocytopenia (platelet count < $50000 /$ cumm) makes a diagnosis of ITP more likely ${ }^{12,14}$ after ruling out other causes for thrombocytopenia. Assessment of individual cases of thrombocytopenia in pregnancy focuses on excluding important underlying causes and weighing risks of bleeding in both mother and baby against hazards of diagnostic and therapeutic interventions. $^{14}$ The reported prevalence of maternal thrombocytopenia is variable. In a recent survey in 2000 involving 6770 pregnant women, prevalence of thrombocytopenia was found to be $11.6 \%$. As there is paucity and wide variations in data regarding prevalence of these causes in Indian women; so the present study was planned out to find prevalence and causes of thrombocytopenia in pregnancy and maternal and neonatal outcome in cases of thrombocytopenia. 


\section{Materials and Methods}

Present study was conducted to identify underlying cause of thrombocytopenia in pregnancy and neonatal outcome of thrombocytopenia in pregnancy.

\section{Inclusion Criteria}

All pregnant women with thrombocytopenia (platelet count $<1 \mathrm{~L} /$ cumm) due to the various causes

\section{Exclusion Criteria}

All pregnant women with normal platelet count.

\section{Study Design}

Hospital Based Prospective Observational Study over a period of two years. A sample of 278 pregnant women cases was selected for study after satisfaction of inclusion and exclusion criteria.

\section{Methodology}

Thrombocytopenic patients were grouped according to the cause of thrombocytopenia. Prevalence of thrombocytopenia and prevalence specific for each cause of thrombocytopenia was calculated. Pregnancy was supervised as per protocol of the hospital and in consultation with the hematologist and decisions regarding admission in hospital, termination of pregnancy, mode of termination were taken accordingly.

Fetal outcome in terms of condition at birth (live/dead), baby's weight, APGAR Score, any bleeding manifestations (like VH, its grade) were noted. Baby's cord blood platelet count was taken to assess neonatal thrombocytopenia and its severity. Thrombocytopenia cases due to different causes were compared in their outcome.

\section{Results}

Out of 278 cases enrolled for study, 50 women were found to have thrombocytopenia, and 228 had normal platelet count. Thus, incidence of thrombocytopenia was $18 \%$.

\section{Causes of Thrombocytopenia}

Various causes of thrombocytopenia in pregnancy found were: Gestational Thrombocytopenia, ITP, HELLP, Dengue and Drug induced thrombocytopenia.

Gestational Thrombocytopenia accounted for majority of cases $(66 \%)$ followed by ITP (12\%). It was further followed by HELLP syndrome (10\%) and dengue $(8 \%)$. One patient was diagnosed to have drug induced thrombocytopenia. Table 1

\section{Birth Weight and Thrombocytopenia}

Out of total, 30\% patients delivered low birth weight babies and $70 \%$ delivered babies with birth weight of $>2.5 \mathrm{~kg}$.

\section{Apgar Score and Thrombocytopenia}

APGAR score (at $1 \mathrm{~min}$ ) of neonates was less than 7 in $12 \%$ newborns while at $5 \mathrm{~min}$ it was below 7 in only $1(2 \%)$ baby.

\section{Neonatal Complications and Thrombocytopenia}

Complications developed in only 3 neonates, one case each of sepsis, ICH and still birth. Table 2

\section{Cord blood platelet count and Thrombocytopenia}

Out of total, $28 \%$ newborns had thrombocytopenia (i.e cord blood platelet count was $<1.0$ lakh /cumm) and $72 \%$ had no thrombocytopenia (i.e. cord blood platelet count $\geq 1.0$ lakh /cumm). Table 3

\section{Birth Weight in Cases of GT and ITP}

Occurrence of low birth weight babies in mothers with GT and ITP were comparable being $27.3 \%$ and $33.3 \%$ respectively (p-0.9). Table 4

\section{Apgar score in cases of GT and ITP}

APGAR score at 1 minute was poor for the neonates born to mothers with ITP in comparison to neonates born to mothers with GT $(\mathrm{p}<0.01)$.

\section{Cord blood platelet count in cases of GT and ITP}

In cases with GT only $6.1 \%$ babies were born with a cord blood platelet count of $<50000 /$ cumm. However in cases with ITP, maximum no of babies $(66.7 \%)$ had cord blood platelet count $<50000 /$ cumm. This data is statistically significant $(\mathrm{p}<0.01)$. Thus, ITP leads to neonatal thrombocytopenia. Table 5.

Table 1: Etiology of Thrombocytopenia

\begin{tabular}{|c|c|c|}
\hline Causes & $\mathbf{N}$ & \% \\
\hline GT & 33 & $66.0 \%$ \\
\hline ITP & 6 & $12.0 \%$ \\
\hline HELLP & 5 & $10.0 \%$ \\
\hline DENGUE & 4 & $8.0 \%$ \\
\hline Drug Induced & 1 & $2.0 \%$ \\
\hline SLE & 1 & $2.0 \%$ \\
\hline Total & 50 & $100.0 \%$ \\
\hline
\end{tabular}

Table 2: Neonatal complications and thrombocytopenia

\begin{tabular}{|c|c|c|}
\hline Neonatal Complications & $\mathbf{N}$ & $\mathbf{\%}$ \\
\hline Still Births & 1 & $2.0 \%$ \\
\hline Sepsis & 1 & $2.0 \%$ \\
\hline ICH & 1 & $2.0 \%$ \\
\hline None & 47 & $94.0 \%$ \\
\hline Total & 50 & $100.0 \%$ \\
\hline
\end{tabular}

Table 3: Cord blood platelet count and thrombocytopenia

\begin{tabular}{|c|c|c|}
\hline Thrombocytopenia (Baby) & $\mathbf{N}$ & $\mathbf{\%}$ \\
\hline Yes & 14 & $28.0 \%$ \\
\hline No & 36 & $72.0 \%$ \\
\hline Total & 50 & $100.0 \%$ \\
\hline
\end{tabular}

Table 4: Birth weight in cases of GT and ITP 


\begin{tabular}{|c|c|c|c|}
\hline \multirow{2}{*}{ Birth Weight } & \multicolumn{2}{|c|}{ Diagnosis } & \multirow{2}{*}{ Total } \\
\cline { 2 - 4 } & GT (n-33) & ITP (n-6) & \\
\hline$<2.5 \mathbf{~ K g}$ & 9 & 2 & 11 \\
\cline { 2 - 4 } & $27.3 \%$ & $33.3 \%$ & $28.2 \%$ \\
\hline \multirow{2}{*}{$>\mathbf{2 . 5} \mathbf{~ K g}$} & 24 & 4 & 28 \\
\cline { 2 - 4 } & $72.7 \%$ & $66.7 \%$ & $71.8 \%$ \\
\hline Total & 33 & 6 & 39 \\
\hline \multicolumn{3}{|c|}{ p- value - 0.9 } \\
\hline
\end{tabular}

Table 5: Cord Blood Platelet Counts In Cases of GT and ITP

\begin{tabular}{|c|c|c|c|}
\hline \multirow{2}{*}{$\begin{array}{l}\text { Cord Blood Platelet } \\
\text { count }\end{array}$} & \multicolumn{2}{|c|}{ Diagnosis } & \multirow[t]{2}{*}{ Total } \\
\hline & GT $(n-33)$ & ITP (n-6) & \\
\hline \multirow[t]{2}{*}{$<50,000$} & 2 & 4 & 6 \\
\hline & $6.1 \%$ & $66.7 \%$ & $15.4 \%$ \\
\hline \multirow[t]{2}{*}{$50,000-1,50,000$} & 18 & 2 & 20 \\
\hline & $54.5 \%$ & $33.3 \%$ & $51.3 \%$ \\
\hline \multirow[t]{2}{*}{$>1,50,000$} & 13 & 0 & 13 \\
\hline & $39.4 \%$ & $0.0 \%$ & $33.3 \%$ \\
\hline Total & 33 & 6 & 39 \\
\hline \multicolumn{4}{|c|}{ p- value $<0.01$} \\
\hline
\end{tabular}

\section{Discussion}

In the present study prevalence of thrombocytopenia came out to be $18.0 \%$. Various causes of thrombocytopenia in pregnancy found were: Gestational Thrombocytopenia (GT), ITP, pre eclampsia and its complications -HELLP, Dengue, SLE and Drug induced thrombocytopenia. Gestational Thrombocytopenia (GT) accounted for majority of cases $(66 \%)$ followed by ITP $(12 \%)$ which was further followed by HELLP syndrome (10\%). Other minor causes were dengue, drug induced thrombocytopenia and autoimmune diseases. Prevalence of Gestational thrombocytopenia was $11.8 \%$. Prevalence of thrombocytopenia as per several earlier done studies; varies from $6 \%$ to $12 \%$. In initial studies by Burrows RF and Kelton $\mathrm{JG}^{2}$ prevalence was reported as around $7.6 \%$. Incidentally detected thrombocytopenia made the largest group $(65.1 \%)$ and the next group of patients $(13.1 \%)$ was composed of healthy women who had some obstetric or medical condition complicating pregnancy. Shehata $\mathrm{N}$ et al ${ }^{1}$ in 1999 prospectively studied different causes of thrombocytopenia in pregnancy with a large sample size. In her study, prevalence of GT among thrombocytopenia was found to be $73.6 \%$ and of preeclampsia/ eclampsia/ HELLP was $21 \%$. Immune disorders of pregnancy accounted for $4.1 \%$ cases while other causes like DIC/ TTP accounted for $1.3 \%$ cases. Boehlen $\mathrm{F}$ et al ${ }^{15,40}$ studied thrombocytopenia in 2000 and again in 2006, and prevalence was reported as $11 \%$ and $6-11 \%$ respectively with GT being the most common cause. In study by SanioS et $\mathrm{al}^{8}$ prevalence was found to be $7.3 \%$. Karim $\mathrm{R}$ et $\mathrm{al}^{6}$ in 2004 reported that thrombocytopenia was observed in $7 \%$ to $10 \%$ pregnancies. According to the study by Mc Crae KR et al in 2010, thrombocytopenia affects 6-10\% of all pregnant females. Singh $\mathrm{N}$ et $\mathrm{al}^{9}$ in 2012 reported prevalence of thrombocytopenia in pregnancy as $8.8 \%$. GT was seen in
$64.2 \%$ cases, obstetric causes were found in $22.1 \%$ cases and medical causes in $13.68 \%$ cases. Hypertensive and hepatic disorders were the most common obstetric causes of thrombocytopenia. ParnasM et al reported the main causes of thrombocytopenia as gestational thrombocytopenia $(59.3 \%)$, ITP $(11.05 \%)$, preeclampsia $(10.05 \%)$, and HELLP syndrome (12.06\%). KhellafM et $\mathrm{al}^{5}$ in 2012 reported that occurrence of thrombocytopenia during pregnancy is frequent and is around 10\% and GT dominates among all causes of thrombocytopenia in pregnancy.

Ozkan $\mathrm{H}$ et al reviewed 29 women with ITP and stated that majority of deliveries were vaginal and none of the neonates had complications attributable to the mode of delivery. Burrows RF and Kelton JG et $\mathrm{al}^{4}$ in 1988 conducted a prospective study for 1 year on a group of women who delivered at McMaster university and demonstrated that gestational thrombocytopenia appears to have no adverse effects on either mothers or their infants. Also, obstetrical interventions like caesarean section because of thrombocytopenia are not justified in these mothers. In a prospective study by Ruggeri $\mathrm{M}$ et al 37 consecutive patients with GT were enrolled, with a total of 41 pregnancies observed. Vaginal delivery carried out in $33 / 41(80 \%)$ whereas 8/41(20\%) had caesarean for obstetric indications. Two patients received blood transfusion for PPH (atonic). Neonatal bleeding didn't occur in any delivery. Webert KE et al in 2003 did retrospective analysis of obstetric patients with ITP. 92 women with ITP were studied. Women in 37 pregnancies $(31.1 \%)$ required treatment to increase platelet counts. Most deliveries $(82.4 \%)$ were vaginal and bleeding at delivery was uncommon. 18 infants $(14.6 \%)$ required treatment for hemostatic impairment with 2 fetal deaths reported. One was due to hemorrhage. ITP in pregnancy carries a low risk, but mothers and infants may require therapy to raise their platelet counts. Suri V et al did a retrospective study in which 16 patients with ITP were studied. There was no postpartum hemorrhage or maternal death. None of the neonates had bleeding complications, irrespective of mode of delivery. They concluded that pregnant patients with ITP have generally good maternal and perinatal outcomes. Singh $\mathrm{N}$ et $\mathrm{al}^{9}$ reported that mode of delivery was not affected by thrombocytopenia. Maternal morbidity and mortality was seen in only medical and obstetric causes of thrombocytopenia.

The present study showed that ITP patients were at a risk of preterm delivery ( $p<0.05$ ) compared to GT patients. There was no difference seen in the occurrence of low birth weight babies in the two groups ( $p>0.05$ ). But neonates born to thrombocytopenic mothers found to have poor 1 minute APGAR scores $(\mathrm{p}<0.01)$. This was supported by Grzyb A et al who reported that premature labor $(<37$ weeks) was observed more often in patients with severe than in moderate thrombocytopenia (6/14-42, $85 \% \mathrm{v} / \mathrm{s} 4 / 31-16$, $13 \%)$. Lower neonatal body weight $(2774.28+/-28$ vs. $3120.32+/-788.22)$, APGAR score in the 1st minute (7.42 $+/-3.56$ vs. $9.13+/-1.56$ ) as well as neonatal complications ( $71.42 \%$ vs. 28.5$)$ were also observed more often in severe 
than in moderate thrombocytopenia group. ParnasM et al did a retrospective case control study in 2006 and reported that the perinatal outcome of GT is favourable but higher rates of labor induction and preterm deliveries, higher rates of intrauterine growth restriction (IUGR), APGAR scores < 7 , still births were noted in women with thrombocytopenia due to rare causes like DIC, familial TTP, APLA syndrome. In our study, there was one still birth in a patient with ITP. Other neonate born to a known case of ITP mother had sepsis and another one had intracranial hemorrhage (after caesarean delivery).

Of the infants born to thrombocytopenic mothers, only $28 \%$ had thrombocytopenia in cord blood. In cases with GT only $6.1 \%$ babies were born with a cord blood platelet count of $<50000$. However in cases with ITP, maximum no of babies $(66.7 \%)$ had cord blood platelet count $<50000$. Thus, ITP leads to neonatal thrombocytopenia $(p=0.000)$. Jenson JD et $\mathrm{al}^{46}$ did a retrospective study in 2011 and linked maternal and neonatal platelet counts. No correlation was observed between the two. In another study, Anteby E et al reported that GT is not accompanied by neonatal thrombocytopenia but ITP is associated with NAIT.

The study demonstrated that thrombocytopenia is a frequent finding in pregnancy. It has many potential causes but gestational thrombocytopenia (GT) predominates. GT was seen to develop in late gestation with normal early gestational counts. Degree of thrombocytopenia was mild to moderate with no adverse effects on either mothers or their infants. Platelet count was seen to resolve within a period of 6 weeks in most cases. In contrast, ITP develops in early gestation with severe degrees of thrombocytopenia with adverse effects on either mothers or their infants.

\section{Conclusion}

This was a prospective observational study conducted in the Department of Obstetrics and Gynaecology of a tertiary care institute. The main objective was to calculate the incidence of thrombocytopenia in pregnant population and to find different causes of thrombocytopenia with their respective prevalence. Different leading causes were compared among themselves in terms of maternal and neonatal outcomes. The prevalence of thrombocytopenia was $18 \%$. Various causes of thrombocytopenia in pregnancy found were: Gestational Thrombocytopenia, ITP, pre eclampsia \& its complications, Dengue, SLE and Drug induced thrombocytopenia. Among patients with thrombocytopenia $20 \%$ had preterm delivery and $80 \%$ had delivery at term. Patients of ITP were at increased risk of preterm delivery $(\mathrm{p}<0.05)$. Out of total, $30 \%$ patients delivered low birth weight babies and $70 \%$ delivered babies with birth wt of $>2.5 \mathrm{~kg}$. Occurrence of low birth weight babies in mothers with GT and ITP were comparable being $27.3 \%$ and $33.3 \%$ respectively (p-0.9). APGAR score (at $1 \mathrm{~min}$ ) of neonates was less than 7 in $12 \%$ neonates while at $5 \mathrm{~min}$ it was below 7 in only 1(2\%) baby. Neonates born to mothers with ITP had poor 1 minute APGAR score when compared to neonates born to patients with GT $(p<0.001)$.There was no significant relationship found between maternal and fetal platelet counts in patients with gestational thrombocytopenia. ITP leads to neonatal thrombocytopenia $(\mathrm{p}<0.01)$. Gestational thrombocytopenia had much favorable perinatal outcome in comparison to ITP.

\section{Conflict of Interest: None.}

\section{References}

1. Shehata N, Burrows RF, Kelton JG. Gestational Thrombocytopenia. Clin Obstet Gynecol 1999;42:327-34.

2. Burrows RF, Kelton JG. Thrombocytopenia at delivery: a prospective survey of 6715 deliveries. Am J Obstet Gynecol 1990;162(3):731-4.

3. Kadir RA, McLintock C. Thrombocytopenia and disorders of platelet function in pregnancy. Semin Thromb Hemost 2011;37(6):640-52.

4. Khellaf M, Loustau V, Bierling P, Michel M, Godeau B. Thrombocytopenia and pregnancy. Rev Med Interne 2012;33(8):446-52.

5. Karim R, Sacher RA. Thrombocytopenia in pregnancy. Curr Hematol Rep 2004;3(2):128-33.

6. Sainio S, Kekomaki R, Rikonen S, Teramo K. Maternal thrombocytopenia at term: a population based study. Acta ObstetGynaecol Scand 2000;79(9):744-9.

7. Singh N, Dhakad A, Singh U, Tripathi, Sankhwar P. Prevalence and Characterization of Thrombocytopenia in Pregnancy in Indian Women. Indian J Hematol Blood Transfus 2012;28:77-81.

8. Boehlen F, Hohlfeld P, Extermann P, Perneger TV, de Moerloose P. Platelet count at term pregnancy: a reappraisal of the threshold. Obstet Gynecol 2000;95(1):29-33.

9. Boehlen F. Thrombocytopenia during Pregnancy. Importance, diagnosis and management. Hemostaseologie 2006;26:72-74

10. Ruggeri M, SchiavottoC, Castaman C, Tosetto A, Rodeghiero F. Gestational thrombocytopenia: A prospective study. Haematologica 1997;82(3):341-2.

11. Webert KE, Mittal R, Sigouin C, Heddle NM, Kelton JG. A retrospective 11-year analysis of obstetric patients with idiopathic thrombocytopenic purpura. Blood 2003;102(13):4306-11.

12. Parnas M, Sheiner E, Shoham-Vardi I, Burstein E, Yermiahu T, Levi I et al. Moderate to Severe Thrombocytopenia during Pregnancy. Eur J Obstet Gynecol Reprod Biol 2006;128:163-8.

13. Ozkan H, Cetinkaya M, Köksal N, Ali R, Güneş AM, Baytan $\mathrm{B}$ et al. Neonatal outcomes of pregnancy complicated by idiopathic thrombocytopenic purpura. $J$ Perinatol 2010;30(1):38-44.

14. Wei J, Liu GL, Liang MY, Wang SM. Effect of general anesthesia used in cesarean section on maternal-neonatal outcome of pregnancy complicated with severe thrombocytopenia. Zhonghua Fu Chan KeZaZhi 2009;44(9):665-8.

How to cite this article: Barsode S, Tarlekar V, Shora M, Mehendale S, Clinical profile of thrombocytopenia in pregnancy. Indian J Obstet Gynecol Res 2019;6(2):192-195. 